\title{
Rainbow Connection Number and Connectivity
}

\author{
Xueliang Li, Sujuan Liu \\ Center for Combinatorics and LPMC-TJKLC \\ Nankai University, Tianjin 300071, China \\ lxl@nankai.edu.cn; sjliu0529@126.com
}

L. Sunil Chandran, Rogers Mathew, Deepak Rajendraprasad

Department of Computer Science and Automation

Indian Institute of Science, Bangalore -560012, India

\{sunil, rogers, deepakr\}@csa.iisc.ernet.in

Submitted: May 31, 2011; Accepted: Oct 12, 2011; Published: Jan 16, 2012

Mathematics Subject Classifications: 05C40, 05C15

\begin{abstract}
The rainbow connection number, $r c(G)$, of a connected graph $G$ is the minimum number of colors needed to color its edges, so that every pair of vertices is connected by at least one path in which no two edges are colored the same. Our main result is that $\operatorname{rc}(G) \leq\left\lceil\frac{n}{2}\right\rceil$ for any 2 -connected graph with at least three vertices. We conjecture that $\operatorname{rc}(G) \leq n / \kappa+C$ for a $\kappa$-connected graph $G$ of order $n$, where $C$ is a constant, and prove the conjecture for certain classes of graphs. We also prove that $r c(G) \leq(2+\varepsilon) n / \kappa+23 / \varepsilon^{2}$ for any $\varepsilon>0$.
\end{abstract}

Keywords: rainbow coloring, rainbow connection number, connectivity, 2-connected graph, ear decomposition, chordal graph, girth

\section{Introduction}

An edge coloring of a graph is a function from its edge set to the set of natural numbers. A path in an edge colored graph with no two edges sharing the same color is called a rainbow path. An edge colored graph is said to be rainbow connected if every pair of vertices is connected by at least one rainbow path. Such a coloring is called a rainbow coloring of the graph. If a rainbow coloring uses $k$ colors, we call it a $k$-rainbow coloring. The minimum number of colors required to rainbow color a connected graph is called

${ }^{*}$ Supported by NSFC No.11071130. 
its rainbow connection number, denoted by $\operatorname{rc}(G)$. For example, the rainbow connection number of a complete graph is 1, that of a path is its length, and that of a star is its number of leaves. For a basic introduction to the topic, see Chapter 11 in [7].

The concept of rainbow coloring was introduced by Chartrand, Johns, McKeon and Zhang [6] in 2008. Chakraborty et al. [4] showed that computing the rainbow connection number of a graph is NP-Hard. To rainbow color a graph, it is enough to ensure that every edge of some spanning tree in the graph gets a distinct color. Hence, the order of the graph minus one is an upper bound for the rainbow connection number. Many authors $[3,4,10]$ view rainbow connection number as a "quantifiable" way of strengthening the connectivity property of a graph. Hence, tighter upper bounds on the rainbow connection number for a graph with higher connectivity have been a subject of investigation.

The following are the results in this direction reported in literature: Let $G$ be a graph of order $n$. Caro et al. [3] showed that if $G$ is 2-edge-connected (bridgeless), then $r c(G) \leq 4 n / 5-1$ and if $G$ is 2-connected, then $r c(G) \leq \min \{2 n / 3, n / 2+O(\sqrt{n})\}$. Li and Shi [11] showed that if $G$ is 3-connected, then $\operatorname{rc}(G) \leq 3(n+1) / 5$. Krivelevich and Yuster [10] showed that $\operatorname{rc}(G) \leq 20 n / \delta$, where $\delta$ is the minimum degree of $G$. The result was improved by Chandran et al. [5] where it was shown that $\operatorname{rc}(G) \leq 3 n /(\delta+1)+3$. Hence it follows that $r c(G) \leq 3 n /(\lambda+1)+3$ if $G$ is $\lambda$-edge-connected and $r c(G) \leq 3 n /(\kappa+1)+3$ if $G$ is $\kappa$-connected. This is because $\kappa \leq \lambda \leq \delta$.

The main result of our paper is that for any 2-connected graph of order $n, \operatorname{rc}(G) \leq$ $\lceil n / 2\rceil$, and the bound is trivially attained by cycles of order at least 4 (Theorem 2.4). This improves the previous best known upper bounds for 2-connected and 3-connected graphs $[3,11]$ mentioned in last paragraph. We show that the bound of $3 n /(\lambda+1)+3$ in terms of the edge-connectivity is tight up to additive constants for infinitely many values of $\lambda$ and $n$ (Example 3.1). We improve the bound for $\kappa$-connected graphs to $r c(G) \leq(2+\epsilon) n / \kappa+23 / \epsilon^{2}$ for any $\epsilon>0$ (Theorem 3.6). We conjecture (Conjecture 3.7) that for a $\kappa$-connected graph $G, r c(G) \leq n / \kappa+C$ where $C$ is a constant. For $\kappa \geq 3$, we show that the conjecture is true for chordal graphs (Theorem 3.11) and graphs of girth at least 7 (Theorem 3.10). It can be easily shown from existing literature that the conjecture is true for all $\kappa$ for some other graph classes like AT-free graphs and circular arc graphs too [5]. We remark that an upper bound of $n / \kappa+C$ will be tight up to additive factors.

All graphs considered in this article are finite, simple and undirected. The length of a path is its number of edges. If $S$ is a subset of vertices of a graph $G$, the subgraph of $G$ induced by the vertices in $S$ is denoted by $G[S]$. The vertex set and edge set of $G$ are denoted by $V(G)$ and $E(G)$, respectively. The order of $G$ (number of vertices) may be denoted by $|G|$.

\section{Result for 2-connected graphs}

At first we study the rainbow connection number of 2-connected graphs. As usual, the term $\kappa$-vertex-connected will be simply addressed as $\kappa$-connected. The following notation 
and terminology are needed in the sequel.

Definition 2.1. Let $F$ be a subgraph of a graph $G$. An ear of $F$ in $G$ is a nontrivial path in $G$ whose endpoints are in $F$ but whose internal vertices are not. A nested sequence of graphs is a sequence $\left(G_{0}, G_{1}, \cdots, G_{k}\right)$ of graphs such that $G_{i} \subset G_{i+1}, 0 \leq i<k$. An ear decomposition of a 2-connected graph $G$ is a nested sequence $\left(G_{0}, G_{1}, \cdots, G_{k}\right)$ of 2-connected subgraphs of $G$ such that: (1) $G_{0}$ is a cycle; (2) $G_{i}=G_{i-1} \bigcup P_{i}$, where $P_{i}$ is an ear of $G_{i-1}$ in $G, 1 \leq i \leq k ;(3) G_{k}=G$.

The next notions are new ones which will play key roles in our proofs.

Definition 2.2. Let $c$ be a $k$-rainbow coloring of a connected graph $G$. If a rainbow path $P$ in $G$ has length $k$, we call $P$ a complete rainbow path; otherwise, it is an incomplete rainbow path. A rainbow coloring $c$ of $G$ is incomplete if for any vertex $u \in V(G), G$ has at most one vertex $v$ such that all the rainbow paths between $u$ and $v$ are complete; otherwise, it is complete.

A complete rainbow path uses all colors of the coloring, while an incomplete rainbow path misses at least one color of the coloring.

For a connected graph $G$, if a spanning subgraph has an (incomplete) $k$-rainbow coloring, then $G$ has an (incomplete) $k$-rainbow coloring. This simple fact will be used in the following proofs.

Lemma 2.1. Let $G$ be a Hamiltonian graph of order $n(n \geq 3)$. Then $G$ has an incomplete $\left\lceil\frac{n}{2}\right\rceil$-rainbow coloring, i.e., $r c(G) \leq\left\lceil\frac{n}{2}\right\rceil$.

Proof. Since $G$ is a Hamiltonian graph, there is a Hamiltonian cycle $C_{n}=v_{1}, v_{2}, \cdots$, $v_{n}, v_{n+1}\left(=v_{1}\right)$ in $G$. Define an edge-coloring $c$ of $C_{n}$ by $c\left(v_{i} v_{i+1}\right)=i$ for $1 \leq i \leq\left\lceil\frac{n}{2}\right\rceil$ and $c\left(v_{i} v_{i+1}\right)=i-\left\lceil\frac{n}{2}\right\rceil$ if $\left\lceil\frac{n}{2}\right\rceil+1 \leq i \leq n$. It is clear that $c$ is a $\left\lceil\frac{n}{2}\right\rceil$-rainbow coloring of $C_{n}$, and the shortest path connecting any two vertices in $V(G)$ on $C_{n}$ is a rainbow path. For any vertex $v_{i}(1 \leq i \leq n)$, only the antipodal vertex of $v_{i}$ has no incomplete rainbow path to $v_{i}$ if $n$ is even. Every pair of vertices in $G$ has an incomplete rainbow path if $n$ is odd. Hence the rainbow coloring $c$ of $C_{n}$ is incomplete. Since $C_{n}$ is a spanning subgraph of $G$, $G$ has an incomplete $\left\lceil\frac{n}{2}\right\rceil$-rainbow coloring.

Let $G$ be a 2 -connected non-Hamiltonian graph of order $n(n \geq 4)$. Then $G$ must have an even cycle. In fact, since $G$ is 2-connected, $G$ must have a cycle $C$. If $C$ is an even cycle, we are done. Otherwise, $C$ is a odd cycle, we then choose an ear $P$ of $C$ such that $V(C) \bigcap V(P)=\{a, b\}$. Since the lengths of the two segments between $a, b$ on $C$ have different parities, $P$ joining with one of the two segments forms an even cycle. Then, starting from an even cycle $G_{0}$, there exists a nonincreasing ear decomposition $\left(G_{0}, G_{1}\right.$, $\left.\cdots, G_{t}, G_{t+1}, \cdots, G_{k}\right)$ of $G$, such that $G_{i}=G_{i-1} \cup P_{i}(1 \leq i \leq k)$ and $P_{i}$ is a longest ear of $G_{i-1}$, i.e., $\ell\left(P_{1}\right) \geq \ell\left(P_{2}\right) \geq \cdots \geq \ell\left(P_{k}\right)$. Suppose that $V\left(P_{i}\right) \cap V\left(G_{i-1}\right)=\left\{a_{i}, b_{i}\right\}(1 \leq$ $i \leq k)$. We call the distinct vertices $a_{i}, b_{i}(1 \leq i \leq k)$ the endpoints of the ear $P_{i}$. Without loss of generality, suppose that $\ell\left(P_{t}\right) \geq 2$ and $\ell\left(P_{t+1}\right)=\cdots=\ell\left(P_{k}\right)=1$. So $G_{t}$ is a 
2-connected spanning subgraph of $G$. Since $G$ is a non-Hamiltonian graph, we have $t \geq 1$. Denote the order of $G_{i}(0 \leq i \leq k)$ by $n_{i}$. All these notations will be used in the sequel.

Lemma 2.2. Let $G$ be a 2-connected non-Hamiltonian graph of order $n(n \geq 4)$. If $G$ has at most one ear with length 2 in the nonincreasing ear decomposition, then $G$ has a incomplete $\left\lceil\frac{n}{2}\right\rceil$-rainbow coloring, i.e., $r c(G) \leq\left\lceil\frac{n}{2}\right\rceil$.

Proof. Since $G_{t}(t \geq 1)$ in the nonincreasing ear decomposition is a 2-connected spanning subgraph of $G$, it only needs to show that $G_{t}$ has an incomplete $\left\lceil\frac{n}{2}\right\rceil$-rainbow coloring. We will apply induction on $t$.

First, consider the case that $t=1$. Let $G$ be a 2-connected non-Hamiltonian graph with $t=1$ in the nonincreasing ear decomposition. The spanning subgraph $G_{1}=G_{0} \bigcup P_{1}$ of $G$ consists of an even cycle $G_{0}$ and an ear $P_{1}$ of $G_{0}$. Without loss of generality, suppose that $G_{0}=v_{1}, v_{2}, \cdots, v_{2 k}, v_{2 k+1}\left(=v_{1}\right)$ where $k \geq 2$. We color the edges of $G_{0}$ with $k$ colors. Define the edge-coloring $c_{0}$ of $G_{0}$ by $c_{0}\left(v_{i} v_{i+1}\right)=i$ for $1 \leq i \leq k$ and $c_{0}\left(v_{i} v_{i+1}\right)=i-k$ if $k+1 \leq i \leq 2 k$. From the proof of Lemma 2.1, the coloring $c_{0}$ is an incomplete $k$-rainbow coloring of $G_{0}$. Now consider $G_{1}$ according to the parity of $\ell\left(P_{1}\right)$. If $\ell\left(P_{1}\right)$ is even, then $n_{1}$ is odd and color the edges of $P_{1}$ with $\frac{\ell\left(P_{1}\right)}{2}$ new colors. In the first $\frac{\ell\left(P_{1}\right)}{2}$ edges of $P_{1}$ the colors are all distinct, and the same ordering of colors is repeated in the last $\frac{\ell\left(P_{1}\right)}{2}$ edges of $P_{1}$. It is easy to verify that the obtained coloring $c_{1}$ of $G_{1}$ is an incomplete $\left\lceil\frac{n_{1}}{2}\right\rceil$-rainbow coloring such that for any pair of vertices in $G$, there exists an incomplete rainbow path connecting them. If $\ell\left(P_{1}\right)$ is odd, then $n_{1}$ is even and color the edges of $P_{1}$ with $\frac{\ell\left(P_{1}\right)-1}{2}$ new colors. The middle edge of $P_{1}$ receives any color that already appeared in $G_{0}$. The first $\frac{\ell\left(P_{1}\right)-1}{2}$ edges of $P_{1}$ all receive distinct new colors and in the last $\frac{\ell\left(P_{1}\right)-1}{2}$ edges of $P_{1}$ this coloring is repeated in the same order. It is easy to verify that the obtained coloring $c_{1}$ of $G_{1}$ is an incomplete $\left\lceil\frac{n}{2}\right\rceil$-rainbow coloring.

Let $G$ be a 2 -connected non-Hamiltonian graph with $t \geq 2$ in the nonincreasing ear decomposition. Assume that the subgraph $G_{i}(1 \leq i \leq t-1)$ has an incomplete $\left\lceil\frac{n_{i}}{2}\right\rceil$ rainbow coloring $c_{i}$ such that when $n_{i}$ is odd, any pair of vertices have an incomplete rainbow path. We distinguish the following three cases.

Case 1. $\ell\left(P_{t}\right)(\geq 3)$ is odd.

Suppose that $P_{t}=v_{0}\left(=a_{t}\right), v_{1}, \cdots, v_{r}, v_{r+1}, \cdots, v_{2 r}, v_{2 r+1}\left(=b_{t}\right)$, where $r \geq 1$. We color the edges of $P_{t}$ with $r$ new colors to obtain an incomplete coloring $c_{t}$ of $G_{t}$. Define an edge-coloring of $P_{t}$ by $c\left(v_{i-1} v_{i}\right)=x_{i}(1 \leq i \leq r), c\left(v_{r} v_{r+1}\right)=x$ and $c\left(v_{i-1} v_{i}\right)=$ $x_{i-r-1}(r+2 \leq i \leq 2 r+1)$, where $x_{1}, x_{2}, \cdots, x_{r}$ are new colors and $x$ is a color appearing in $G_{t-1}$. It is easy to check that the obtained coloring $c_{t}$ of $G_{t}$ is a $\left\lceil\frac{n}{2}\right\rceil$-rainbow coloring.

Now we show that $c_{t}$ is incomplete such that when $n_{t}$ is odd, any pair of vertices have an incomplete rainbow path. For any pair of vertices $u, v \in V\left(G_{t-1}\right) \times V\left(G_{t-1}\right)$, the rainbow path $P$ from $u$ to $v$ in $G_{t-1}$ is incomplete in $G_{t}$, because the new colors $x_{1}, x_{2}, \cdots, x_{r}(r \geq 1)$ do not appear in $P$. For any pair of vertices $u, v \in V\left(P_{t}\right) \times V\left(P_{t}\right)$, if there exists a rainbow path $P$ from $u$ to $v$ on $P_{t}$, then $P$ is incomplete in $G_{t}$, since some color in $G_{t-1}$ does not appear in $P$; if not, there exists an incomplete rainbow path $P$ from $u$ to $v$ through some vertices of $G_{t-1}$ such that at least one new color does not 
appear in $P$. For any pair of vertices $u, v \in V\left(G_{t-1}\right) \times\left(V\left(P_{t}\right) \backslash\left\{v_{r}, v_{r+1}\right\}\right)$, there exists an incomplete rainbow path from $u$ to $v$ in which at least one new color does not appear. If there exists a vertex all of whose rainbow paths to $a_{t}$ (resp. $b_{t}$ ) in $G_{t-1}$ are complete, we denote the vertex by $a_{t}^{\prime}$ (resp. $\left.b_{t}^{\prime}\right)$. For vertex $v_{r}$ (resp. $\left.v_{r+1}\right)$, only the vertex $a_{t}^{\prime}$ (resp. $b_{t}^{\prime}$ ) possibly has no incomplete rainbow path to $v_{r}$ (resp. $v_{r+1}$ ) in $G_{t}$. So there possibly exist two pairs of vertices $a_{t}^{\prime}, v_{r}$ and $b_{t}^{\prime}, v_{r+1}$ which have no incomplete rainbow path. Since $a_{t}^{\prime}, b_{t}^{\prime}$ are distinct in $G_{t-1}$, the rainbow coloring $c_{t}$ is incomplete. If $n$ is odd, then $n_{t-1}$ is odd. By induction, $a_{t}^{\prime}$, $b_{t}^{\prime}$ do not exist when $n_{t-1}$ is odd. Hence every pair of vertices have a incomplete rainbow path.

Case 2. $\ell\left(P_{t}\right)(\geq 2)$ is even and $n_{t-1}$ is even.

In this case, $n$ is odd. Suppose that $P_{t}=v_{0}\left(=a_{t}\right), v_{1}, \cdots, v_{r}, v_{r+1}, \cdots, v_{2 r-1}, v_{2 r}(=$ $\left.b_{t}\right)$, where $r \geq 1$. Define an edge-coloring of $P_{t}$ by $c\left(v_{i-1} v_{i}\right)=x_{i}$ for $1 \leq i \leq r$ and $c\left(v_{i-1} v_{i}\right)=x_{i-r}$ for $r+1 \leq i \leq 2 r$. It is clear that the obtained coloring $c_{t}$ of $G_{t}$ is a $\left\lceil\frac{n}{2}\right\rceil$-rainbow coloring.

Now we prove that $c_{t}$ is incomplete such that when $n_{t}$ is odd, any pair of vertices have an incomplete rainbow path. For any pair of vertices in $V\left(G_{t-1}\right) \times V\left(G_{t-1}\right)$ or $V\left(P_{t}\right) \times V\left(P_{t}\right)$, there is an incomplete rainbow path connecting them in $G_{t}$, similar to the Case 1. For any pair of vertices $u \in V\left(G_{t-1}\right), v \in V\left(P_{t}\right)\left(v \neq v_{r}\right)$, there is an incomplete rainbow path $P$ from $u$ to $v$ such that at least one new color does not appear in $P$. For any vertex $u \in V\left(G_{t-1}\right)$, since the coloring $c_{t-1}$ is incomplete, $u$ has an incomplete rainbow path $P^{\prime}$ in $G_{t-1}$ to one of $a_{t}, b_{t}$ (say $a_{t}$ ). Then $P^{\prime}$ joining with $a_{t} P_{t} v_{r}$ is an incomplete rainbow path from $u$ to $v_{r}$ in $G_{t}$. Therefore, the rainbow coloring $c_{t}$ of $G_{t}$ is incomplete such that any pair of vertices has an incomplete rainbow path.

Case 3. $\ell\left(P_{t}\right)(\geq 2)$ is even and $n_{t-1}$ is odd.

In this case, $n$ is even. We consider the following three subcases.

Subcase 3.1. $\left[V\left(P_{t}\right) \bigcap V\left(P_{t-1}\right)\right] \backslash V\left(G_{t-2}\right)=\emptyset$.

If $\ell\left(P_{t-1}\right)$ is odd, let $G_{t-1}^{\prime}=G_{t-2} \bigcup P_{t}$ and $G_{t}=G_{t-1}^{\prime} \cup P_{t-1}$. By induction, $G_{t-1}^{\prime}$ has an incomplete $\left\lceil\frac{n_{t-1}^{\prime}}{2}\right\rceil$-rainbow coloring $\left(n_{t-1}^{\prime}\right.$ is the order of $\left.G_{t-1}^{\prime}\right)$. Similar to Case 1, we can obtain an incomplete $\left\lceil\frac{n}{2}\right\rceil$-rainbow coloring of $G_{t}$ from $G_{t-1}^{\prime}$.

Suppose that $\ell\left(P_{t-1}\right)$ is even. By induction, $G_{t-2}$ has an incomplete $\left\lceil\frac{n_{t-2}}{2}\right\rceil$-rainbow coloring $c_{t-2}$. Suppose that $P_{t-1}=v_{0}\left(=a_{t-1}\right), v_{1}, \cdots, v_{r}, v_{r+1}, \cdots, v_{2 r-1}, v_{2 r}\left(=b_{t-1}\right)$ and $P_{t}=v_{0}^{\prime}\left(=a_{t}\right), v_{1}^{\prime}, \cdots, v_{s}^{\prime}, v_{s+1}^{\prime}, \cdots, v_{2 s-1}^{\prime}, v_{2 s}^{\prime}\left(=b_{t}\right)$, where $r \geq 2, s \geq 1$. Since $c_{t-2}$ is incomplete and $a_{i}, b_{i}(1 \leq i \leq k)$ are two distinct vertices, then $a_{t-1}$ has an incomplete rainbow path $P^{\prime}$ to one of $a_{t}, b_{t}$ (say $a_{t}$ ) and $b_{t-1}$ has an incomplete rainbow path $P^{\prime \prime}$ to the other vertex. Suppose that $x$ is the color in $G_{t-2}$ that does not appear in $P^{\prime}$. Now color the edges of $P_{t-1}, P_{t}$ with $r+s-1$ new colors and the color $x$. Define an edge-coloring of $P_{t-1}$ by $c\left(v_{i-1} v_{i}\right)=x_{i}(1 \leq i \leq r)$ and $c\left(v_{i-1} v_{i}\right)=x_{i-r}(r+1 \leq i \leq 2 r)$, where $x_{1}, x_{2}, \cdots, x_{r}$ are new colors. Define an edge-coloring of $P_{t}$ by $c\left(v_{i-1}^{\prime} v_{i}^{\prime}\right)=y_{i}(1 \leq$ $i \leq s-1), c\left(v_{s-1}^{\prime} v_{s}^{\prime}\right)=x, c\left(v_{s}^{\prime} v_{s+1}^{\prime}\right)=x_{1}$ and $c\left(v_{i-1}^{\prime} v_{i}^{\prime}\right)=y_{i-s-1}(s+2 \leq i \leq 2 s)$, where $y_{1}, y_{2}, \cdots, y_{s-1}$ are new colors.

Similar to Case 2 , the obtained coloring $c_{t-1}$ of $G_{t-1}$ is an incomplete $\left\lceil\frac{n_{t-1}}{2}\right\rceil$-rainbow 
coloring such that every pair of vertices have an incomplete rainbow path. It is obvious that $G_{t}$ is rainbow connected. The path $\left(v_{s}^{\prime} P_{t} a_{t}\right) P^{\prime}\left(a_{t-1} P_{t-1} v_{r}\right)$ is a rainbow path from $v_{s}^{\prime}$ to $v_{r}$ which is possibly complete. For any other pair of vertices in $G_{t}$, there is an incomplete rainbow path connecting them. Hence the rainbow coloring $c_{t}$ of $G_{t}$ is incomplete.

Subcase 3.2. $\left[V\left(P_{t}\right) \bigcap V\left(P_{t-1}\right)\right] \backslash V\left(G_{t-2}\right)=\left\{b_{t}\right\}$.

If $\ell\left(P_{t-1}\right)$ is odd, suppose that $P_{t-1}=v_{0}\left(=a_{t-1}\right), v_{1}, \cdots, v_{r}, v_{r+1}, \cdots, v_{2 r}, v_{2 r+1}(=$ $\left.b_{t-1}\right)$. Since $P_{t-1}$ is a longest ear of $G_{t-2}$ and $b_{t} \in V\left(P_{t-1}\right) \backslash V\left(G_{t-2}\right)$, we have $r \geq$ 2. Define an edge-coloring of $P_{t-1}$ by $c\left(v_{i-1} v_{i}\right)=x_{i}(1 \leq i \leq r), c\left(v_{r} v_{r+1}\right)=x$ and $c\left(v_{i-1} v_{i}\right)=x_{i-r-1}(r+2 \leq i \leq 2 r+1)$, where $x_{1}, x_{2}, \cdots, x_{r}$ are new colors and $x$ is a color appearing in $G_{t-2}$. Similar to Case 1, the obtained coloring $c_{t-1}$ of $G_{t-1}$ is an incomplete $\left\lceil\frac{n_{t-1}}{2}\right\rceil$-rainbow coloring such that every pair of vertices have an incomplete rainbow path. If $\ell\left(P_{t-1}\right)$ is even, suppose that $P_{t-1}=v_{0}\left(=a_{t-1}\right), v_{1}, \cdots, v_{r}, v_{r+1}, \cdots, v_{2 r-1}, v_{2 r}\left(=b_{t-1}\right)$, where $r \geq 2$. Define an edge-coloring of $P_{t-1}$ by $c\left(v_{i-1} v_{i}\right)=x_{i}(1 \leq i \leq r)$, and $c\left(v_{i-1} v_{i}\right)=x_{i-r}(r+1 \leq i \leq 2 r)$, where $x_{1}, x_{2}, \cdots, x_{r}$ are new colors. Similar to Case 2 , the obtained coloring $c_{t-1}$ of $G_{t-1}$ is an incomplete $\left\lceil\frac{n_{t-1}}{2}\right\rceil$-rainbow coloring such that every pair of vertices have an incomplete rainbow path.

Without loss of generality, assume that $b_{t}$ belongs to the first half of $P_{t-1}$ and that $P_{t}=v_{0}^{\prime}\left(=a_{t}\right), v_{1}^{\prime}, \cdots, v_{s}^{\prime}, v_{s+1}^{\prime}, \cdots, v_{2 s-1}^{\prime}, v_{2 s}^{\prime}\left(=b_{t}\right)$, where $s \geq 1$. We color the edges of $P_{t}$ with $s-1$ new colors. Define an edge-coloring of $P_{t}$ by $c\left(v_{i-1}^{\prime} v_{i}^{\prime}\right)=y_{i}(1 \leq i \leq s-1)$, $c\left(v_{s-1}^{\prime} v_{s}^{\prime}\right)=x_{1}, c\left(v_{s}^{\prime} v_{s+1}^{\prime}\right)=y$ and $c\left(v_{i-1}^{\prime} v_{i}^{\prime}\right)=y_{i-s-1}(s+2 \leq i \leq 2 s)$, where $y_{1}, y_{2}, \cdots, y_{s-1}$ are new colors, $y$ is a color in $G_{t-2}$ and $x \neq y$. It is easy to verify that the obtained coloring $c_{t}$ of $G_{t}$ is a $\left\lceil\frac{n}{2}\right\rceil$-rainbow coloring.

For any pair of vertices $v^{\prime} \in V\left(P_{t}\right)\left(v^{\prime} \neq v_{s}^{\prime}\right)$ and $v \in V\left(G_{t-1}\right)$, there exists an incomplete rainbow path $P$ connecting them, since the path from $v^{\prime}$ to the nearest endpoint of $P_{t}$ joining with the incomplete rainbow path from the endpoint to $v$ in $V\left(G_{t-1}\right)$ is an incomplete rainbow path from $v^{\prime}$ to $v$ in $G_{t}$. For $v_{s}^{\prime}$, there is an incomplete rainbow path from $v_{s}^{\prime}$ to any vertex in $V\left(G_{t-2}\right) \cup V\left(b_{t-1} P_{t-1} v_{r+2}\right)$ through edge $e=v_{s-1}^{\prime} v_{s}^{\prime}$, and an incomplete rainbow path from $v_{s}^{\prime}$ to any vertex in $V\left(a_{t-1} P_{t-1} v_{r+1}\right)$ through edge $e=v_{s}^{\prime} v_{s+1}^{\prime}$. For any pair of vertices in $V\left(P_{t}\right) \times V\left(P_{t}\right)$, there is an incomplete rainbow path connecting them. Hence the rainbow coloring $c_{t}$ is incomplete.

Subcase 3.3. $\left[V\left(P_{t}\right) \bigcap V\left(P_{t-1}\right)\right] \backslash V\left(G_{t-2}\right)=\left\{a_{t}, b_{t}\right\}$.

We can prove this subcase in a way similar to Subcase 3.2. Without loss of generality, we can assume that $a_{t}=v_{p}(1 \leq p \leq r-1)$ and $b_{t}=v_{q}(q \geq p+2)$. Color all the edges of $P_{t-1}$ and $P_{t}$ as in Subcase 3.2 but only the edge $e=v_{s-1}^{\prime} v_{s}^{\prime}$ which is colored by $x_{p+1}$ in $P_{t-1}$ instead. The obtained coloring $c_{t}$ of $G_{t}$ is an incomplete $\left\lceil\frac{n}{2}\right\rceil$-rainbow coloring.

Lemma 2.3. Let $G$ be a 2-connected non-Hamiltonian graph of order $n(n \geq 4)$. If $G$ has at least 2 ears of length 2 in the nonincreaing ear decomposition, then $\operatorname{rc}(G) \leq\left\lceil\frac{n}{2}\right\rceil$.

Proof. We only need to prove that there exists a rainbow coloring $c_{t}$ of the spanning subgraph $G_{t}$ in the nonincreasing ear decomposition that uses at most $\left\lceil\frac{n}{2}\right\rceil$ colors. If $G$ has 2 or 3 ears of length 2 in the nonincreaing ear decomposition, then $G_{t-2}$ has at most one ear of length 2 and $\ell\left(P_{t-1}\right)=\ell\left(P_{t}\right)=2$. From Lemmas 2.1 and $2.2, G_{t-2}$ 
has an incomplete $\left\lceil\frac{n_{t-2}}{2}\right\rceil$-rainbow coloring $c_{t-2}$. Assume that $P_{t-1}=a_{t-1}, v, b_{t-1}$ and $P_{t}=a_{t}, v^{\prime}, b_{t}$. Since $P_{t-1}$ is a longest ear of $G_{t-2}$, we have $a_{t}, b_{t} \in V\left(G_{t-2}\right)$. Since the coloring $c_{t-2}$ is incomplete, $a_{t-1}$ has an incomplete rainbow path $P$ to one of $a_{t}, b_{t}$ (say $a_{t}$ ) such that the color $x$ in $G_{t-2}$ does not appear in $P$. Define an edge-coloring of $P_{t-1}$ and $P_{t}$ by $c\left(a_{t-1} v\right)=c\left(b_{t-1} v\right)=c\left(b_{t} v^{\prime}\right)=x_{1}$ and $c\left(a_{t} v^{\prime}\right)=x$, where $x_{1}$ is a new color. It is clear that $v a_{t-1} P a_{t} v^{\prime}$ is a rainbow path from $v$ to $v^{\prime}$, and the obtained coloring of $G_{t}$ is a $\left\lceil\frac{n}{2}\right\rceil$-rainbow coloring.

Now consider the case that $G$ has at least 4 ears of length 2 in the nonincreaing ear decomposition. Suppose that $\ell\left(P_{t^{\prime}-1}\right) \geq 3$ and $\ell\left(P_{t^{\prime}}\right)=\ell\left(P_{t^{\prime}+1}\right)=\cdots=\ell\left(P_{t}\right)=2$. Since $P_{i}(1 \leq i \leq k)$ is a longest ear of $G_{i-1}$, we have that $a_{t^{\prime}}, b_{t^{\prime}}, \cdots, a_{t}, b_{t} \in V\left(G_{t^{\prime}-1}\right)$, i.e., $V\left(G_{t^{\prime}-1}\right)$ is a connected 1-step dominating set. From Lemmas 2.1 and 2.2, there exists a $\left\lceil\frac{n_{t^{\prime}-1}}{2}\right\rceil$-rainbow coloring $c_{t^{\prime}-1}$ of $G_{t^{\prime}-1}$. Color one edge of $P_{i}\left(t^{\prime} \leq i \leq t\right)$ with $x_{1}$ and the other with $x_{2}$, where $x_{1}, x_{2}$ are two new colors. It is obvious that $G_{t}$ is rainbow connected. Since $G$ has at least 4 ears of length 2 , the obtained rainbow coloring of $G_{t}$ uses at most $\left\lceil\frac{n}{2}\right\rceil$ colors.

From the above three lemmas and the fact that $r c\left(C_{n}\right)=\left\lceil\frac{n}{2}\right\rceil(n \geq 4)$, we can derive our following main result.

Theorem 2.4. Let $G$ be a 2-connected graph of order $n(n \geq 3)$. Then $\operatorname{rc}(G) \leq\left\lceil\frac{n}{2}\right\rceil$, and the upper bound is tight for $n \geq 4$.

Since for any two distinct vertices in a $\kappa$-connected graph $G$ of order $n$, there exist at least $\kappa$ internal disjoint paths connecting them, the diameter of $G$ is no more than $\left\lfloor\frac{n}{\kappa}\right\rfloor$. One could think of generalizing Theorem 2.4 to the case of higher connectivity. Therefore we conjecture that for any $\kappa$-connected graph $G, r c(G) \leq\left\lceil\frac{n}{\kappa}\right\rceil$.

\section{$3 \quad$ Results for graphs with higher connectivity}

Now we will deal with graphs with higher (edge-) connectivity. At first, we settle the question of a tight upper bound for rainbow connection number in terms of edgeconnectivity by showing that the bound of $3 n /(\lambda+1)+3$, which directly follows from the minimum degree bound of $3 n /(\delta+1)+3[5]$, is tight up to additive factors. We show the tightness by constructing a family of $\lambda$-edge-connected graphs for infinitely many values of $\lambda$ and order $n$ with diameter $d=\frac{3 n}{\lambda+1}-3$. Since diameter is a lower bound on the rainbow connection number, the construction suffices for our purpose.

Example 3.1 (Construction of a $\lambda$-edge-connected graph $G$ on $n$ vertices with diameter $\left.d=\frac{3 n}{\lambda+1}-3\right)$. Let $d \geq 1$ be a natural number, and $\lambda$ a natural number such that $\lambda+1$ is a multiple of 3 and $\lambda \geq 8$. Set $k:=\frac{\lambda+1}{3}$, and set $V(G)=V_{0} \uplus V_{1} \uplus \cdots \uplus V_{d}$, where $\left|V_{i}\right|$ is $2 k$ for $i=0$ and $i=d$, and $k$ for $1 \leq i<d$. Two distinct vertices $u \in V_{i}$ and $v \in V_{j}$ are adjacent in $G$ if and only if $|i-j| \leq 1$. It is easy to see that the diameter of $G$ is $d, n=|V(G)|=k(d+3)$ and hence $d=\frac{n}{k}-3=\frac{3 n}{\lambda+1}-3$. By considering all pairs of vertices, it can be seen that $G$ is $\lambda$-edge-connected. 
Next, we try to obtain an upper bound on the rainbow connection number for $\kappa$ connected graphs that is tighter than the $3 n /(\kappa+1)$ bound implied by the degree bound in [5]. We will show that for any $\kappa$-connected graph $G, \operatorname{rc}(G) \leq(2+\epsilon) n / \kappa+23 / \epsilon^{2}$ for any $\epsilon>0$. The following notation and terminology are needed.

Definition 3.1. Given a graph $G$, a set $D \subseteq V(G)$ is called an $\ell$-step dominating set of $G$, if every vertex in $G$ is at a distance at most $\ell$ from $D$. Furthermore, if $G[D]$ is connected, then $D$ is called a connected $\ell$-step dominating set of $G$.

From [1] we have the following lemma.

Lemma 3.2 ([1]). If $G$ is a bridgeless graph, then for every connected $\ell$-step dominating set $D^{\ell}$ of $G, \ell \geq 1$, there exists a connected $(\ell-1)$-step dominating set $D^{\ell-1} \supset D^{\ell}$ such that

$$
r c\left(G\left[D^{\ell-1}\right]\right) \leq r c\left(G\left[D^{\ell}\right]\right)+2 \ell+1 .
$$

The following three lemmas are used to prove our theorem.

Lemma 3.3. If $G$ is a bridgeless graph, and $D^{\ell}$ is a connected $\ell$-step dominating set of $G$, then

$$
r c(G) \leq r c\left(G\left[D^{\ell}\right]\right)+\ell(\ell+2) \leq\left|D^{\ell}\right|-1+\ell(\ell+2) .
$$

Proof. Note that the only 0-step dominating set in $G$ is $V(G)$. Hence the first inequality follows from repeated application of Lemma 3.2. The second inequality follows since $\operatorname{rc}\left(G\left[D^{\ell}\right]\right) \leq\left|D^{\ell}\right|-1$.

Lemma 3.4. Every $\kappa$-connected $(\kappa \geq 1)$ graph $G$ of order $n$ has a connected $2 \ell$-step dominating set of size at most $\left(\frac{2 \ell+1}{\kappa \ell+1}\right) n$ for every natural number $\ell \geq 0$.

Proof. If $k \leq 2$, the bound is trivial for any $\ell \geq 0$ since we can take $V(G)$ as the dominating set. Similarly, if $r$ is the radius of $G$, for $\ell \geq r / 2$ we can take any central vertex of $G$ as the $2 \ell$-step dominating set. Hence we assume $\kappa>2$ and $\ell<r / 2$.

The following procedure is used to construct a $2 \ell$-step dominating set $D$. Let $N^{i}(S):=$ $\left\{x: d_{G}(x, S)=i\right\}$ and $\overline{N^{i}}(S):=\left\{x: d_{G}(x, S) \leq i\right\}$ for any $S \subset V(G) . N^{i}(s)=N^{i}(\{s\})$ and $\overline{N^{i}}(s)=\overline{N^{i}}(\{s\})$ for any $s \in V(G)$.

$D=\{u\}$, for some $u \in V(G)$.

While $N^{2 \ell+1}(D) \neq \emptyset$,

\{

Pick any $v \in N^{2 \ell+1}(D)$. Let $\left(v, x_{2 \ell}, x_{2 \ell-1}, \ldots, x_{0}\right), x_{0} \in D$ be a shortest $v-D$ path.

\} 
Clearly, $D$ remains connected after every iteration of the procedure. Since the procedure ends only when $N^{2 \ell+1}(D)=\emptyset$, the final $D$ is a $2 \ell$-step dominating set. Let $t$ be the number of iterations executed by the procedure. From Menger Theorem (see [2] for example) we know that when the procedure starts $\left|\overline{N^{\ell}}(D)\right|=\left|\overline{N^{\ell}}(u)\right| \geq \kappa \ell+1$. This is because $\ell<r$ and $\left|N^{i}(u)\right| \geq \kappa$ for $1 \leq i<r$ since $G$ is $\kappa$-connected. Note that $v \in N^{2 \ell+1}(D)$ ensures that $\overline{N^{\ell}}(v) \cap \overline{N^{\ell}}(D)=\emptyset$, and $\left|\overline{N^{\ell}}(v)\right| \geq \kappa \ell+1$ due to $\kappa$-connectivity of $G$. Hence the addition of $v$ to $D$ increases $\left|\overline{N^{\ell}}(D)\right|$ by at least $\kappa \ell+1$ in every iteration. Therefore, when the procedure ends, $(\kappa \ell+1)(t+1) \leq\left|\overline{N^{\ell}}(D)\right| \leq n$. Since $D$ starts as a singleton set and each iteration adds $2 \ell+1$ more vertices, $|D|=(2 \ell+1) t+1 \leq \frac{(2 \ell+1) n}{\kappa \ell+1}-2 \ell \leq\left(\frac{2 \ell+1}{\kappa \ell+1}\right) n$.

Lemma 3.5. If $G$ is a $\kappa$-connected $(\kappa \geq 1)$ graph of order $n$, then for every natural number $\ell \geq 0$,

$$
r c(G) \leq\left(\frac{2 \ell+1}{\kappa \ell+1}\right) n+2 \ell(2 \ell+2)-1 .
$$

Proof. The case $\kappa=1$ is trivial. Hence we assume $\kappa \geq 2$ and therefore $G$ is bridgeless. Since $G$ is $\kappa$-connected, by Lemma 3.4 , for every $\ell \geq 0$ we have a $2 \ell$-step dominating set $D$ of size at most $\left(\frac{2 \ell+1}{\kappa \ell+1}\right) n$. Now an application of Lemma 3.3 gives the result.

Theorem 3.6. For every $\kappa \geq 1$, if $G$ is a $\kappa$-connected graph of order $n$, then for every $\epsilon \in(0,1)$,

$$
r c(G) \leq\left(\frac{2+\epsilon}{\kappa}\right) n+\frac{23}{\epsilon^{2}}
$$

Proof. Given an $\epsilon \in(0,1)$, choose $\ell=\left\lceil\frac{1}{\epsilon}\right\rceil$. Then the result follows from Lemma 3.5. Note that $2 \ell(2 \ell+2)-1 \leq 23 / \epsilon^{2}$.

The above bound may not be tight, and we are tempted to believe that the following conjecture might be true.

Conjecture 3.7. For every $\kappa \geq 1$, if $G$ is a $\kappa$-connected graph of order $n$, then $r c(G) \leq$ $n / \kappa+C$, where $C$ is a constant.

Now we show some cases in which Conjecture 3.7 is true, namely high girth graphs (where the girth of a graph is the size of a shortest cycle in the graph, denoted by girth $(G)$ ), chordal graphs (where a graph is called chordal if it contains no induced cycles of length greater than 3). We mainly consider $\kappa$-connected graphs with $\kappa \geq 3$, since for 2-connected graphs we have shown in above section that the conjecture is true, and the upper bound is tight.

Lemma 3.8. Every connected graph $G$ of order $n$, minimum degree $\delta \geq 3$ and girth at least $2 g+1$ has a connected $2 g$-step dominating set of size at most $\left(\frac{2 g+1}{C_{\delta, g}}\right) n-2 g$, where $C_{\delta, g}=\frac{\delta(\delta-1)^{g}-2}{\delta-2}$. 
Proof. Note that $\delta \geq 3$ ensures that $G$ is not a tree and hence the girth is finite, that is, $1 \leq g<\infty$. Now observe that for any vertex $v \in V(G),\left|\overline{N^{g}}(\{v\})\right| \geq 1+\delta+\delta(\delta-1)+$ $\ldots+\delta(\delta-1)^{g-1}$. The summation on the right hand side is equal to $C_{\delta, g}$, for $\delta \geq 3$. Now the proof follows the same steps as in that of Lemma 3.4 after setting $\ell=g$. Hence we omit the details.

Lemma 3.9. Let $G$ be a connected graph of minimum degree $\delta$. Then,

1. if $\delta \geq 3$ and $\operatorname{girth}(G) \geq 7$, then $r c(G)<n / \delta+41$ and

2. if $\delta \geq 5$ and $\operatorname{girth}(G) \geq 5$, then $r c(G)<n / \delta+19$.

Proof. For the first result, from substituting the mentioned values of minimum degree and girth in Lemma 3.8 and then by applying Lemma 3.3 we get that $r c(G) \leq \frac{7}{C_{\delta, 3}} n-$ $6-1+6 \times 8=\frac{7}{C_{\delta, 3}} n+41=\frac{n}{\delta} \cdot \frac{7 \delta(\delta-2)}{\delta(\delta-1)^{3}-2}+41 \leq \frac{n}{\delta} \cdot \frac{21}{22}+41<\frac{n}{\delta}+41$, in which we used the monotonicity of the function $f(\delta)=\frac{7 \delta(\delta-2)}{\delta(\delta-1)^{3}-2}$ for the second inequality " $\leq$ ". The proof for the second result is similar.

Since vertex connectivity of a graph is a lower bound for minimum degree, the following results is immediate.

Theorem 3.10. Let $G$ be a $\kappa$-connected graph. Then,

1. if $\kappa \geq 3$ and $\operatorname{girth}(G) \geq 7$, then $\operatorname{rc}(G)<n / \kappa+41$ and

2. if $\kappa \geq 5$ and $\operatorname{girth}(G) \geq 5$, then $r c(G)<n / \kappa+19$.

Theorem 3.11. For every $\kappa$-connected chordal graph $G$ of order $n$,

$$
r c(G) \leq \frac{n}{\kappa}+3
$$

Proof. The case of $\kappa=1$ is trivial since rainbow coloring a spanning tree of $G$ suffices. Hence let us assume $\kappa \geq 2$ and hence $G$ is bridgeless. We claim that $G$ has a 1-step connected dominating set $D$ which can be rainbow colored using $\frac{|D|}{\kappa}$ colors. Then by Lemma 3.3, $r c(G)-3 \leq r c(D) \leq \frac{|D|}{\kappa} \leq \frac{n}{\kappa}$. Hence it remains to prove the above claim. Consider a maximal connected set $D \subset V(G)$ that can be rainbow colored using $\frac{|D|}{\kappa}$ colors. Such a set exists since any singleton set of vertices can be rainbow colored using $0<\frac{1}{\kappa}$ colors. Suppose for contradiction that $D$ is not a 1-step dominating set. Then $N_{G}(D)$ is a vertex separator and hence contains a minimal separator $S \subset N_{G}(D)$. Since $G$ is $\kappa$ connected, $|S| \geq \kappa$, and since $G$ is chordal, $S$ induces a clique [8]. Giving a single new color to every $D-S$ and $S-S$ edge extends the rainbow coloring of $G[D]$ to $G[D \cup S]$. Thus $D \cup S$ is a connected set containing $D$ which can be colored using $r c(G[D])+1 \leq \frac{|D|}{\kappa}+1 \leq \frac{|D \cup S|}{\kappa}$ colors, contradicting the maximality of $D$. So $D$ is a 1-step connected dominating set and thus the result follows.

Acknowledgement: The authors would like to thank the referee for helpful comments and suggestions. 


\section{References}

[1] M. Basavaraju, L.S. Chandran, D. Rajendraprasad, A. Ramaswamy, Rainbow connection number and radius, arXiv: 1011.0620v1 [math.CO] 2010.

[2] J.A. Bondy, U.S.R. Murty, Graph Theory, GTM 244, Springer, 2008.

[3] Y. Caro, A. Lev, Y. Roditty, Z. Tuza, and R. Yuster, On rainbow connection, Electron. J. Combin. 15(1)(2008), R57.

[4] S. Chakraborty, E. Fischer, A. Matsliah, R. Yuster, Hardness and algorithms for rainbow connection, 26th International Symposium on Theoretical Aspects of Computer Science STACS 2009(2009), 243-254. Also, see J. Combin. Optim. 21(2011), 330-347.

[5] L.S. Chandran, A. Das, D. Rajendraprasad, N.M. Varma, Rainbow connection number and connected dominating sets, arXiv : 1010.2296v1 [math.CO] 2010.

[6] G. Chartrand, G.L. Johns, K.A. McKeon, P. Zhang, Rainbow connection in graphs, Math. Bohem, 133(1)(2008), 85-98.

[7] G. Chartrand, P. Zhang, Chromatic Graph Theory, Chapman \& Hall, 2008.

[8] G. Dirac, On rigid circuit graphs, Abhandlungen aus dem Mathematischen Seminar der Universitt Hamburg, 25(1961), 71-76. 10.1007/BF02992776.

[9] J. Dong, X. Li, Rainbow connection number, bridges and radius, arXiv : 1105.0790v1 [math.CO] 2011.

[10] M. Krivelevich, R. Yuster, The rainbow connection of a graph is (at most) reciprocal to its minimum degree, J. Graph Theory, 63(3)(2010), 185-191.

[11] X. Li, Y. Shi, Rainbow connection in 3-connected graphs, arXiv : 1010.6131v1 [math.CO] 2010.

[12] I. Schiermeyer, Rainbow connection in graphs with minimum degree three, In J. Fiala, J. Kratochvl, M. Miller, Ed., Combinatorial Algorithms, Lecture Notes in Computer Science Vol.5874 (2009), 432-437. Springer Berlin/Heidelberg. 